\title{
Confined LO phonon limited free carrier absorption in quantum well wires
}

\author{
G.B. Ibragimov ${ }^{1}$, Derin Hüseyin ${ }^{2}$, Yaraneri Halil ${ }^{2}$ \\ ${ }^{I}$ Institute of Physics, National Academy of Sciences of the Azerbayijan Republic, \\ Prosp. H. Javid, 33, Baku, Az 1143, Azerbayijan \\ ${ }^{2}$ Adnan Menderes University, 09010-Aydin, Turkey \\ E-mail: guseyn@physics.ab.az;guseyn_gb@mail.ru
}

\begin{abstract}
The theory of free-carrier absorption is given for quantum well wire for the case where the carriers are scattered by confined longitudinal-optical (LO) phonons and the radiation field is polarized along the length of the wire. Both the multisubband structure and confined phonon modes are considered together. The free carrier absorption coefficients are calculated taking into account all possible LO phonon modes as well as all possible electron intersubband transitions. The results show that the absorption coefficient decreases with increasing the photon frequency and decreases with temperature. It was found that in quantum wire the electron-optic phonon interaction give a greater contribution to the absorption than the electron-confined LO phonon interaction. The results are interpreted in terms of confined LO phonons-assisted transitions between size quantized subbands.
\end{abstract}

Keywords: quantum well wires, confined longitudinal-optical, free-carrier absorption.

Manuscript received 11.04.06; accepted for publication 23.10.06.

\section{Introduction}

With the developments in epitaxial techniques, it is possible to fabricate quasi-one-dimensional (Q1D) semiconductor quantum well wires (QWWs) of various geometries. The motion of electrons in such semiconducting structures is confined and leads to size quantization effects which play an important role in determining their optical and electronic properties. Owing to the limited number of available final states during a scattering process, these structures will exhibit high mobilities [1] and are potentially important for high-speed device applications. Scattering mechanisms due to various processes have been studied theoretically and experimentally [2-8].

Free-carrier absorption (FCA) [9-12], which occurs concurrently with intersubband absorption, greatly distorts the absorption spectra, especially in the long-wavelength regions [13], since the free-carrier absorption coefficients are proportional to the carrier density and, roughly, to the square of the wavelength of the involved photons. Moreover, the FCA effect often hides the peak position of intersubband absorption spectra, which means the peak absorption wavelength (transition energy of the intersubband absorption) cannot be obtained solely from experi- mental absorption spectra $[10,13]$. Therefore, to design a practical infrared detector with a specific peak absorption wavelength, it is necessary to distinguish between intersubband absorption and free-carrier absorption.

FCA is also very important in certain other devices, especially in long-wavelength semiconductor lasers. In semiconductor lasers, the refractive index change includes an anomalous dispersion component and the free-carrier component due to the plasma effect (freecarrier absorption) [14]. Therefore, the FCA can give rise to a change in carrier-induced refractive index, which affects the characteristics of semiconductor lasers (particularly long-wavelength lasers), such as spectral linewidth and wavelength chirping [14]. It has been reported that FCA in the case of quantum well lasers increases the linewidth enhancement factor by about $40 \%$ [15]. For carriers confined in a quantum wells, the FCA is practically important for determining the optical absorption. Scattering-assisted absorption by free electrons and holes in the active quantum wells then usually determines the internal loss in optically pumped laser devices with undoped claddings. Even in electrically pumped devices, assisted FCA can dominate if the lasing mode is optically confined primarily to the active region, as in the interband cascade lasers [16]. 
FCA in diode optical cladding layers consisting of superlattice injectors [17] can also be significant.

Since FCA is one of the powerful means to understand the scattering mechanisms of carriers, it has been studied in QWWs, theoretically, for the case absorption assisted by acoustic [18], acoustic and optical phonons [19], piezoelectric coupling, acoustic and optical phonons [20], interface-roughness [21] and alloydisorder [22]. However, confined longitudinal-optical (LO) phonon scattering is an important scattering mechanism in QWWs and some quantum-mechanical studies have been performed [23-27].

In this paper, we present the theory of FCA for the Q1D electron gas in QWW structures when carriers are scattered by confined LO phonons. We consider the FCA for the cases where the radiation field is polarized along the length of the wire. Absorption coefficient will be calculated for the examples of GaAs QWWs.

\section{Formalism}

We assume that a gas of carriers is confined to move in a long thin wire that is embedded in an insulating cladding. For simplicity, we choose the cross section of the wire to be rectangular with $L_{z}$ and $L_{y}$ the cross sectional dimensions along the $z$ and $y$ directions, respectively, and with $L$ the wire length along the $x$ direction where electrons are assumed to move freely. Assuming the usual effective-mass approximation for the conduction band, the energy eigenfunctions and eigenvalues for electrons in a rectangular thin wire can be written as

$$
\begin{aligned}
& E_{k j l}=E_{k}+E_{j}+E_{l}=\frac{\hbar^{2} k^{2}}{2 m^{*}}+j^{2} E_{a}^{0}+l^{2} E_{b}^{0}, \\
& E_{a}^{0}=\frac{\pi^{2} \hbar^{2}}{2 m^{*} L_{y}^{2}}, E_{b}^{0}=\frac{\pi^{2} \hbar^{2}}{2 m^{*} L_{z}^{2}}, j, l=1,2,3, \ldots, \\
& \Psi_{k j l}=\left[2 /\left(L_{y} L_{z} L\right)^{1 / 2}\right] \sin \left(\pi j y / L_{y}\right) \times \\
& \times \sin \left(\pi l z / L_{z}\right) \exp (i k x) .
\end{aligned}
$$

The FCA coefficient $\alpha$, which is related to the quantum-mechanical transition probabilities in which the carriers absorb or emit a photon with the simultaneous scattering of the carriers from phonons, is given by $\alpha=\frac{\varepsilon_{0}^{1 / 2}}{n_{0} c} \sum_{i} W_{i} f_{i}$.

Here $\varepsilon_{0}$ is the static dielectric constant of material, $n_{0}$ is the number of photons in the radiation field and $f_{i}$ is the free carrier distribution function. The sum is over all the possible initial states $i$ of the system. The transition probabilities $W_{i}$ can be calculated using the standard second-order Born golden rule approximation:

$$
\begin{aligned}
& W_{i}=\frac{2 \pi}{\hbar} \sum_{f q}\left[\left|\left\langle f\left|M_{+}\right| i\right\rangle\right|^{2} \delta\left(E_{f}-E_{i}-\hbar \Omega-\hbar \omega_{q}\right)+\right. \\
& \left.+\left|\left\langle f\left|M_{-}\right| i\right\rangle\right|^{2} \delta\left(E_{f}-E_{i}-\hbar \Omega+\hbar \omega_{q}\right)\right] .
\end{aligned}
$$

Here $E_{i}$ and $E_{f}$ are the initial and final state energies, respectively, of electrons, $\hbar \Omega$ is the photon energy, $\hbar \omega_{q}$ is the phonon energy, and $\left\langle f\left|M_{ \pm}\right| i\right\rangle$ are the transition matrix elements from the initial state to the final state for the interaction between electrons, photons and phonons. This transition matrix element can be represented by

$$
\begin{aligned}
& \left\langle f\left|M_{ \pm}\right| i\right\rangle=\sum_{\alpha}\left(\frac{\left\langle f\left|H_{R}\right| \alpha\right\rangle\left\langle\alpha\left|H_{\mathrm{LO}}\right| i\right\rangle}{E_{i}-E_{\alpha} \mp \hbar \omega_{q}}+\right. \\
& \left.+\frac{\left\langle f\left|H_{\mathrm{LO}}\right| \alpha\right\rangle\left\langle\alpha\left|H_{R}\right| i\right\rangle}{E_{i}-E_{\alpha}-\hbar \Omega}\right),
\end{aligned}
$$

where $H_{R}$ is the interaction Hamiltonian between the electrons and the radiation field, $H_{\mathrm{LO}}$ is the scattering potential due to the electron-phonon interaction.

The interaction Hamiltonian with the radiation field is

$$
H_{R}=-\frac{e}{m^{*}}\left(\frac{2 \pi \hbar n_{0}}{V \Omega \varepsilon_{0}}\right)^{1 / 2}(\varepsilon P),
$$

where $\varepsilon$ is the polarization vector of the radiation field, $V$ is the volume of the crystal, and $P$ is the electron momentum.

The interaction of electrons with the confined-LO phonon is given by [23]

$$
\begin{aligned}
H_{\mathrm{LO}}= & 2 \alpha^{\prime} \sum_{k_{x}} e^{i k_{x} x}\left\{\sum_{m=1,3,5, \ldots} \sum_{n=1,3,5, \ldots} \frac{\cos \left(m \pi y / L_{y}\right) \cos \left(n \pi z / L_{z}\right)}{\left[k_{x}^{2}+\left(m \pi / L_{y}\right)^{2}+\left(n \pi / L_{z}\right)^{2}\right] 1 / 2}\left[A_{\mathrm{LO}}\left(k_{x}\right)+A_{\mathrm{LO}}{ }^{+}\left(-k_{x}\right)\right]+\right. \\
& +\sum_{\mathrm{m}=1,3,5, \ldots} \sum_{n=2,4,6, \ldots} \frac{\cos \left(m \pi y / L_{y}\right) \sin \left(n \pi z / L_{z}\right)}{\left[k_{x}^{2}+\left(m \pi / L_{y}\right)^{2}+\left(n \pi / L_{z}\right)^{2}\right]^{1 / 2}}\left[A_{\mathrm{LO}}\left(k_{x}\right)+A_{\mathrm{LO}}{ }^{+}\left(-k_{x}\right)\right]
\end{aligned}
$$


where $A_{\mathrm{LO}}\left(k_{x}\right)$ and $A_{\mathrm{LO}}\left(-k_{x}\right)$ are the annihilation and creation operators for appropriate LO-phonon modes of the QWW.

$$
\alpha^{\prime}=\left\{\left(e^{2} / 2 \varepsilon_{0} L_{y} L_{z} L\right) \hbar \omega_{\mathrm{LO}}\left[1 / \varepsilon_{\infty}-1 / \varepsilon_{0}\right]\right\}^{1 / 2},
$$

where $\varepsilon_{\infty}$ is the optical dielectric constant or the highfrequency dielectric constant of the material.

Using Eq. (3) and straightforward calculation of transition probabilities using matrix elements of $H_{R}$ and $H_{\mathrm{LO}}$, we obtain the following expression for the FCA coefficient in a Q1D semiconducting structure:

$\alpha=\frac{e^{4} \omega_{L O}}{4 \pi^{2} \varepsilon^{3 / 2} c \hbar^{3} \Omega^{3} L_{y} L_{z}} \sum_{j l} \sum_{j l^{\prime}} \int f\left(E_{k n l}\right) \times$

$\times\left\{N_{q}\left[E_{k}-2 \sqrt{E_{k}\left(E_{k}+Z_{+}\right)}+\left(E_{k}+Z_{+}\right)\right] I\left(q_{+a}, L_{y}, L_{z}\right)+\right.$

$+\left[E_{k}+2 \sqrt{E_{k}\left(E_{k}+Z_{+}\right)}+\left(E_{k}+Z_{+}\right)\right] \times$

$\left.\times I\left(q_{-a}, L_{y}, L_{z}\right)\right) / \sqrt{E_{k}\left(E_{k}+Z_{+}\right)}+\left(N_{q}+1\right) \times$

$\times \frac{\left[E_{k}+2 \sqrt{E_{k}\left(E_{k}+Z_{-}\right)}+\left(E_{k}+Z_{-}\right)\right] I\left(q_{+e}, L_{y}, L_{z}\right)}{\sqrt{E_{k}\left(E_{k}+Z_{-}\right)}}+\left(N_{q}+1\right) \times$

$\left.\times \frac{\left[E_{k}-2 \sqrt{E_{k}\left(E_{k}+Z_{-}\right)}+\left(E_{k}+Z_{-}\right)\right] I\left(q_{-e}, L_{y}, L_{z}\right)}{\sqrt{E_{k}\left(E_{k}+Z_{-}\right)}}\right\} d E_{k}$.

Here, $\quad Z_{ \pm}=E_{j l}-E_{j^{\prime} l^{\prime}}+\hbar \Omega \pm \hbar \omega, \quad q_{ \pm a}=-k \pm$ $\pm \sqrt{Z_{+} 2 m} / \hbar, \quad q_{ \pm e}=k \pm \sqrt{Z_{-} 2 m} / \hbar, n_{q}$ is the number of phonons in a made of wave vector $q$ with the BoseEinstein distribution function. Moreover

$$
\begin{aligned}
& I_{\mathrm{LO}}\left(k_{x}, L_{y}, L_{z}\right)=\left(\frac{1}{\varepsilon_{1}(\infty)}-\frac{1}{\varepsilon_{1}(0)}\right) \frac{(2 \pi)^{2}}{L_{y} L_{z}} \times \\
& \times \sum_{\mathrm{m}=1,2,3 \ldots n=1,2,3, \ldots}\left\{\frac{4 P_{m n}}{\left[k_{x}^{2}+\left(m \pi / L_{y}\right)^{2}+\left(n \pi / L_{z}\right)^{2}\right]^{1 / 2}}\right\}^{2}
\end{aligned}
$$

with

$$
\begin{aligned}
& P_{m n}=\int_{0}^{L_{y}} d y \frac{2}{L_{y}} \int_{0}^{L_{z}} d z \frac{2}{L_{z}} \sin \left(\frac{j \pi y}{L_{y}}\right) \sin \left(\frac{j^{\prime} \pi y}{L_{y}}\right) \times \\
& \times \sin \left(\frac{l \pi z}{L_{z}}\right) \sin \left(\frac{l^{\prime} \pi z}{L_{z}}\right) \sin \left(\frac{m \pi y}{L_{y}}\right) \sin \left(\frac{n \pi z}{L_{z}}\right) .
\end{aligned}
$$

In the quantum limit, in which only the $j=l=1$ ground level is occupied, the dominant contribution to the sum over phonon modes is made by the mode with $m=n=1$, Eq. (9) may be approximated as [23]

$$
\begin{aligned}
& I_{\mathrm{LO}}\left(k_{x}, L_{y}, L_{z}\right)=\left(\frac{1}{\varepsilon_{\infty}}-\frac{1}{\varepsilon_{0}}\right) \frac{(2 \pi)^{2}}{L_{y} L_{z}} \times \\
& \times \frac{16(8 / 3 \pi)^{4}}{k^{2}+\left(\pi / L_{y}\right)^{2}+\left(\pi / L_{z}\right)^{2}} .
\end{aligned}
$$

For the case of a nondegeneration, Q1D electron gas, the electron distribution function is

$$
\begin{aligned}
& f_{k j l}=\frac{(2 \pi)^{1 / 2 \hbar n_{e} a b}}{\gamma \delta\left(m^{*} K_{\mathrm{B}} T\right)^{1 / 2}} \exp \left[-\frac{j^{2} E_{a}^{0}+l^{2} E_{b}^{0}}{K_{\mathrm{B}} T}\right] \times \\
& \times \exp \left(-\frac{\hbar^{2} k^{2}}{2 m^{*} K_{\mathrm{B}} T}\right), \\
& \gamma=\sum_{j} \exp \left(-\frac{j^{2} E_{a}^{0}}{K_{\mathrm{B}} T}\right), \quad \delta=\sum_{l} \exp \left(-\frac{l^{2} E_{b}^{0}}{K_{\mathrm{B}} T}\right),
\end{aligned}
$$

where $n_{e}$ is the concentration of the electrons. Using Eq. (10) in Eq. (7) in the quantum limit we obtain the FCA coefficient

$$
\begin{aligned}
& \alpha=\frac{3 \pi^{1 / 2}(8 / 3 \pi)^{5} e^{4} \omega_{\mathrm{LO}} n_{e}}{\sqrt{2} m^{* 3 / 2} \varepsilon_{0}^{3 / 2} \Omega^{3} c L_{y} L_{z}\left(K_{\mathrm{B}} T\right)^{1 / 2}}\left(\frac{1}{\varepsilon_{\infty}}-\frac{1}{\varepsilon_{0}}\right) \times \\
& \times \sum_{j l} \sum_{j^{\prime} l^{\prime \prime}}\left\{N_{q} \exp \left(\frac{Z_{+}}{2 K_{\mathrm{B}} T}\right) K_{0}\left(\frac{Z_{+}}{2 K_{\mathrm{B}} T}\right)+\right. \\
& +\left(N_{q}+1\right) \exp \left(\frac{Z_{-}}{2 K_{\mathrm{B}} T}\right) K_{0}\left(\frac{Z_{-}}{2 K_{\mathrm{B}} T}\right)- \\
& \left.-N_{q} \frac{\left(b^{2}-Z_{+}^{2}\right)}{\left(K_{\mathrm{B}} T\right)^{2}} F\left(Z_{+}\right)-\left(N_{q}+1\right) \frac{\left(b^{2}-Z_{+}^{2}\right)}{\left(K_{\mathrm{B}} T\right)^{2}} F\left(Z_{-}\right)\right\} .
\end{aligned}
$$

Here, $K_{n}(x)$ is the modified Bessel function of the second kind of argument $x$ and order $n$, and

$$
\begin{aligned}
& b=\frac{\hbar^{2} \pi^{2}}{2 m^{*} L_{y}^{2}}+\frac{\hbar^{2} \pi^{2}}{2 m^{*} L_{z}^{2}}, \\
& F\left(Z_{ \pm}\right)= \\
& =\int_{0}^{\infty} \frac{e^{-x} d x}{\sqrt{x\left(x+Z_{ \pm} / K_{\mathrm{B}} T\right)}\left(4 b x / K_{\mathrm{B}} T+\left(Z_{ \pm}+b\right)^{2} /\left(K_{\mathrm{B}} T\right)^{2}\right)} .
\end{aligned}
$$

\section{Discussion}

We have obtained general expressions FCA for the quantum wires when the carriers are scattered by confined LO phonons. The FCA coefficient is expressed as a function of the photon energy $(\hbar \Omega)$, cross section wire $\left(L_{y} L_{z}\right)$ and temperature $T$. We have evaluated, numerically, the above expressions for FCA coefficient at 300 and $77 \mathrm{~K}$, and parameters characteristic of $\mathrm{GaAs}$ and electron concentration $n_{e}=10^{17} \mathrm{~cm}^{-3}$, phonon energy $\hbar \omega_{\mathrm{LO}}=36.2 \mathrm{meV}$. 


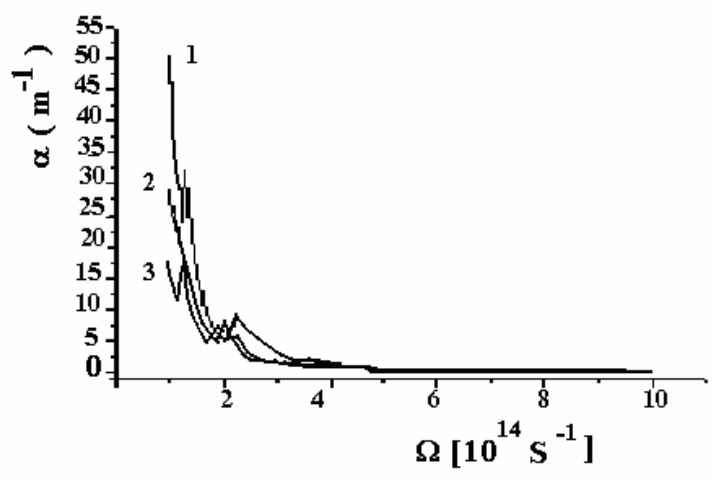

Fig. 1. FCA coefficient in the GaAs QWWs for confined LO phonon scattering as a function of the photon frequency with $T=300 \mathrm{~K}$. We have chosen: $L_{y}=L_{z}=10^{-6} \mathrm{~cm}(1) ; L_{y}=$ $2 \cdot 10^{-6} \mathrm{~cm}, L_{z}=10^{-6} \mathrm{~cm}(2) ; L_{y}=L_{z}=2 \cdot 10^{-6} \mathrm{~cm}, L_{y}=L_{z}=$ $10^{-6} \mathrm{~cm}, L_{y}=L_{z}=2 \cdot 10^{-6} \mathrm{~cm}, L_{y}=L_{z}=10^{-6} \mathrm{~cm}(3)$.

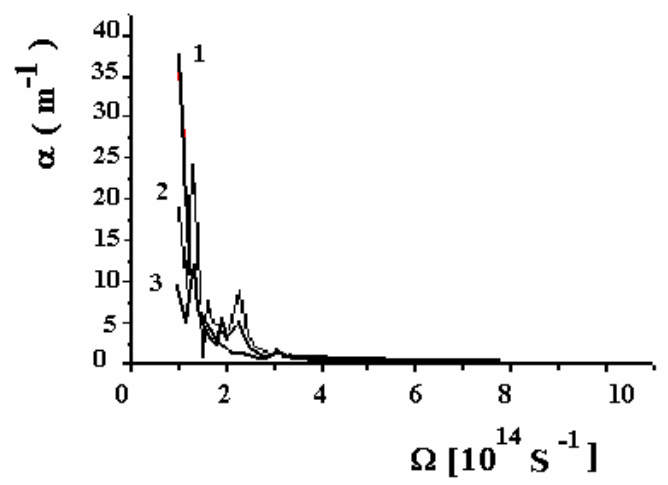

Fig. 2. FCA coefficient in the GaAs QWWs for confined LO phonon scattering as a function of the photon frequency with $T=77 \mathrm{~K}$, the values of $L_{y}, L_{z}$ are the same as in Fig. 1 .

In Figs 1 and 2, we plot the FCA coefficient as a function of the photon frequency $\Omega$ in GaAs QWW with the temperature $T=300 \mathrm{~K}$ and $77 \mathrm{~K}$. From Figs 1 and 2 it is shown that FCA coefficient decreases monotonically with increases with temperature. It is shown that FCA coefficient is an oscillatory function of the photon frequency in this case. These oscillations can be understood in terms of the subband structure, which arises because of the confinement of the carriers. When the photon energy is greater than the energy separation between the various subbands, the absorption of a photon can occur with the simultaneous emission or absorption of optical phonons in transitions to the same or to other subbands. The kinks in the curves indicate confined LO phonon-assisted transition between the subbands. There is a kink in the absorption coefficient as a function of photon frequency $\Omega$ followed by peak. The absorption coefficient is found to be considerably enhanced as the cross-sectional area of the wire decreases. It was predicted [23] that the scattering of electrons by confined LO phonons in QWWs increases as the transverse dimensions of the wire diminish. From Fig. 1 and Ref. [19], it is shown that in quantum wire the electron-optic phonon interaction give a greater contribution to the absorption than the electron-confined LO phonon interaction.

\section{Acknowledgements}

This work was supported by the TUBITAK NATO-PC Advanced Fellowship Programm.

\section{References}

1. H. Sakaki, Scattering suppression and highmobility effect of size-quantized electrons in ultrafine semiconductor wire structures // Jpn J. Appl. Phys. 19(12), p. L735-1739 (1980).

2. V.K. Arora, Size-dependent electric conductivity in semiconducting thin wires // Phys. status solidi (b) 105, p. 707-713 (1981).

3. J. Lee and M.O. Vassel, Low-field electron transport in quasi-one-dimensional semiconducting structures // J. Phys. C: Solid State Phys. 17, p. 2525-2535 (1984).

4. G. Fishman, Phonon-limited mobility in a quasione-dimensional semiconductor // Phys. Rev. B 36(14), p. 7448-7455 (1987).

5. C.C. Wu and C.J. Lin, Impurity-limited mobility of semiconducting thin wires in n-type gallium arsenide // J. Appl. Phys. 83(3), p. 1390-1395 (1988).

6. G.B. Ibragimov, Alloy scattering in quantum well wire structures of semiconductor ternaries // Semiconductor Physics, Quantum Electronics \& Optoelectronics 5, p. 347-352 (2002).

7. S. Kundu, C.K. Sakar and P.K. Basu, Low-field mobility and thermopower in one-dimensional electron gas // J. Appl. Phys. 68(3), p. 1070-1074 (1990).

8. J.W. Brown and H.N. Spector, Impurity scattering limited momentum relaxation time in a quantum well wire // J. Vac. Sci. Technol. B 4(2), p. 453-458 (1986).

9. H.Y. Fan, W. Spitzer and R.J. Colins, Infrared absorption in n-type germanium // Phys. Rev. 101, p. 566-572 (1956).

10. J.S. Park, R.P.G. Karunasiri and K.L. Wang, Normal incidence infrared detector using p-type $\mathrm{SiGe} / \mathrm{Si}$ multiple quantum wells // Appl. Phys. Lett. 60, p. 103-105 (1992).

11. C.Y. Tsai, C.H. Chen, T.L. Sung, T.Y. Wu and F.P. Shih, Theoretical model for intravalley and intervalley free-carrier absorption in semiconductor lasers: Beyond the classical Drude model // IEEE J. Quantum Electron. 34, p. 552-558 (1998).

12. C.C. $\mathrm{Wu}$, C.J. Lin, Free-carrier absorption in heavily doped quasi-two-dimensional semiconducting structures // Phys. Low-dim. Struct. 1/2, p. 281-286 (1998). 
13. C. Lee, Intersubband absorption in conduction bands of silicon and germanium wells. Ph.D. dissertation, University of California, Los Angeles (1994).

14. S. Murata, A. Tomita and A. Suziki, Influence of free carrier plasma effect on carrier - induced refractive index change for quantum-well lasers // IEEE Photon. Technol. Lett. 5, p. 16-19 (1993).

15. L.F. Tiemeijer, P.J.A. Thijs, J.J.M. Binsma, and T.V. Dongen, Effect of the free carriers on the line with enhancement factor of InGaAs/InP (strainedlayer) multiple quantum well lasers // Appl. Phys. Lett. 60, p. 2466-2468 (1992).

16. L.J. Olafsen, E.H. Aifer, I. Vurgaftman, W.W. Benley, C.L. Felix, D. Zhang, C.H. Lin and S.S. Pei, Near-room temperature mid-infrared interband cascade laser // Ibid. 72, p. 2370 (1998).

17. W.W. Bewley, E.H. Aifer, C.L. Felix, I. Vurgaftman, J.R. Meyer, C. H. Lin, S.J. Murry, D. Zhang and S.S. Pei // Ibid. 71, p. 3607 (1997).

18. S.S. Kubakaddi and B.G. Mulimani, Free carrier absorption in semiconducting quantum well wires // J. Phys. C: State Phys. 18, p. 6647-6652 (1985).

19. H. Adamska and N. Spector, Free-carrier absorption from electrons in confined systems // J. Appl. Phys. 59, p. 619-626 (1986).

20. Wu Chhi-Chong, Lin Chau-Jy, Effect of electronphonon scattering mechanisms on free-carrier absorption in quasi-one-dimensional structures // Physica B 316-317, p. 346-349 (2002).
21. G.B. Ibragimov, Theory of the free-carrier absorption in quantum wires with boundary roughness scattering // Semiconductor Physics, Quantum Electronics \& Optoelectronics 6, p. 9-13 (2003).

22. G.B. Ibragimov Free-carrier absorption in semiconducting quantum well wire for alloydisorder scattering // J. Phys.: Condens. Matter 14, p. 8145-8152 (2002).

23. K.W. Kim, M.A. Stroscio, A Bhatt, R Mickevicius and V.V. Mitin, Electron-optical-phonon scattering rates in a rectangular semiconductor quantum wire // J. Appl. Phys. 70, p.319-327 (1991).

24. K Chang, R.Z. Wang, B.K. Ma, The effect of transverse electric field on the electro-opticalphonon scattering rates in quantum wires // Physica $B$ 229, p.347-353 (1996).

25. R. Mickevicius, V. Mitin, G. Paulavicius, V. Kochelap, M.A. Stroscio and G.J. Iafrate, Hotphonon effects on electron transport in quantum wires // J. Appl. Phys. 80, p. 5145-5149 (1996).

26. C.R. Bennett, N.C. Constantinou, M. Babiker and B.K. Ridley, The interaction of electrons with optical phonons in embedded circular and elliptical GaAs quantum wires // J. Phys.: Condens. Matter 7, p. 9819-9832 (1995).

27. J. Pozela, K. Pozela, and V. Jucine, Electron mobility and electron scattering by polar optical phonons in heterostructure quantum wire // Fizika, tekhnika poluprovod. 34, p. 1053-1057 (2000) (in Russian). 